\title{
$\phi$-Meson Photoproduction with Linearly Polarized Photons at Threshold Energies
}

\author{
Julian Salamanca, Philip L. Cole, and the CLAS Collaboration \\ Idaho State University, Dept. of Physics, Pocatello, Idaho 83209 USA
}

\begin{abstract}
The observables provided by linearly-polarized photons are of interest in delineating the contributions of the various hadronic processes giving rise to vector meson photoproduction. In particular, we describe how $\phi$-meson production affords an incisive tool for exploring the nature of the parity exchange at threshold energies, the strangeness content of proton, as well as extracting signatures for the violation of Okubo-Zweig-Iizuka observation (OZI rule). Our goal is to study the $\vec{\gamma} p \rightarrow \phi p$ reaction, with $\phi \rightarrow K^{+} K^{-}$, in the photon energy range of 1.7 to $2.1 \mathrm{GeV}$ by using the Coherent Linear Bremsstrahlung Facility in Hall B of Jefferson Laboratory, Newport News, VA. The data were collected during the g8b run in the summer of 2005.
\end{abstract}

Keywords: photoproduction of $\phi$-mesons, linearly-polarized photons, polarization observables PACS: 14.40.Ev, 25.20.Lj, 29.27. $\mathrm{Hj}$

\section{INTRODUCTION}

At high energies, the diffractive scattering of vector mesons is predominantly mediated by pomeron exchange and not by quark annihilation. However, as one approaches energies within the baryon resonance regime of $2.0 \mathrm{GeV}$, pure pomeron exchange does not provide a complete description of $\phi$-meson production. Moreover, near threshold, one finds quark-exchange in the $t$ channel becomes a key factor. The Okubo-Zweig-Iizuka observation, or OZI rule, suppresses meson exchange in the $t$ channel. Yet the branching ratio of $\phi \rightarrow \rho \pi$ decay is about $15.3 \%$. This brings into question how perfect the OZI suppression rule really is [1]. And if not perfect, the degree of OZI suppression may well provide insight into the nature of the parity exchange in the $t$-channel exchange as measured in Ref. [2]. In Ref. [1], R.A. Williams made two calculations within the same model differing only in the treatment of the $\phi$ production threshold behavior of the pomeron processes. The extraction of the spin density matrix elements as a function of momentum transfer squared, $t$, is the incisive tool for disentangling the mix of pomeron $\left(0^{+}\right.$natural parity) and pseudo-scalar meson exchange $(\pi \eta$ unnatural parity) at low $t$ within the Vector Dominance Model (VDM). At higher $t$ any departure from VDM predictions will reflect additional non-diffractive OZI evading processes such as $s \bar{s}$ knockout $[3,4]$ or resonance production mechanisms $[5,6]$.

\section{THE G8B EXPERIMENT: LAYOUT AND MEASUREMENT}

We seek to extract polarization observables from the reaction $\vec{\gamma} p \rightarrow p \phi \rightarrow K^{+} K^{-}$[7]. The experiment took place in Hall B of Jefferson Laboratory in the summer of 2005 during the $\mathrm{g} 8 \mathrm{~b}$ run. The Coherent Bremsstrahlung Facility, CBF, provided the linearlypolarized photon beam and is depicted in Fig. 1. Electrons from the Continuous Electron

CP947, VII Latin American Symposium on Nuclear Physics and Applications

edited by R. Alarcon, P. L. Cole, C. Djalali, and F. Umeres

(C) 2007 American Institute of Physics 978-0-7354-0461-8/07/\$23.00 


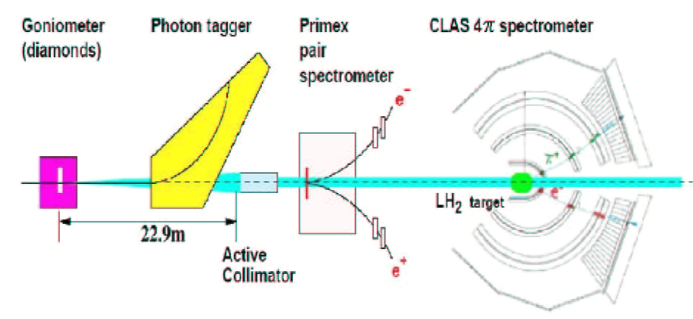

FIGURE 1. CLAS with the Coherent Bremsstrahlung Facility Beamline.

Beam Accelerator Facility (CEBAF) are directed upon a wafer-thin diamond radiator, which produce coherent bremsstrahlung photons [8]. The CBF provided a well monitored beam of linearly-polarized photons with average polarizations of $75 \%$ for energies between 1.7 and $2.1 \mathrm{GeV}$. The beam was tagged to within $\Delta E=0.1 \%$ and was highly collimated to one-half of a characteristic angle (or $\sim 50 \mu \mathrm{rad}$ ) and was continuously monitored online by both the active collimator and the PrimEx pair spectrometer. This tight collimation reduced the incoherent Bethe-Heitler photon background by a factor of five, thereby enhancing the coherent peak and consequently increasing the polarization as shown in Fig. 2. Downstream of the CBF, the photon beam impinges upon a liquid

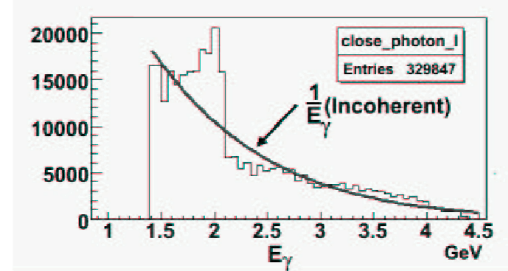

a

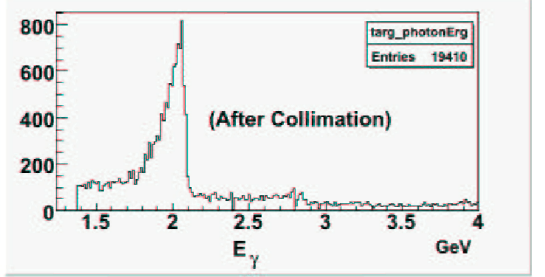

b

FIGURE 2. a) Photon spectrum from coherent bremsstrahlung before collimation. b) Photon spectrum after collimation to $1 / 2$ of a characteristic angle. The degree of polarization is between 70 and $80 \%$.

hydrogen target, which is placed $20 \mathrm{~cm}$ upstream of the geometric center of the CEBAF Large Acceptance Spectrometer or CLAS. CLAS spans $70 \%$ of $4 \pi$ and the sub-detectors inside CLAS, coupled with the toroidal magnetic field, allow for identifying the finalstate particles in high-multiplicity events [9]. Pions, kaons, and protons can readily be identified as seen in Fig. 3b, after all $\beta$, momentum and timing calculations. Measuring the angular distribution of the pseudo-scalar meson decay products, $K^{+}$and $K^{-}$, in the rest frame of the $\phi$ meson is the key for disentangling the various $\phi$ photoproduction mechanisms, i.e. $\vec{\gamma} p \rightarrow p \phi \rightarrow p K^{+} K^{-}$. This angular distribution can be expressed as a linear combination of three functions, $W^{0}, W^{1}$, and $W^{2}$, which in turn are parameterized in terms of the nine spin density matrix elements [10]. And since $W^{1}$ and $W^{2}$ are not measurable with unpolarized photon beams, linearly-polarization gives access to six more density matrix elements over just the three from the $W^{0}$ term. The lower indices of $\rho_{\alpha \beta}^{n}, \alpha, \beta=-1,0,1$, correspond to the possible helicity states of the vector meson [12], 
and $\left|\rho_{\alpha \beta}^{n}\right| \leq 1$

$$
W(\cos \theta, \phi, \Phi)=W^{0}\left(\cos \theta, \phi, \rho_{\alpha \beta}^{0}\right)-P_{\gamma} \cos 2 \Phi W^{1}\left(\cos \theta, \phi, \rho_{\alpha \beta}^{1}\right)-P_{\gamma} \sin 2 \Phi W^{2}\left(\cos \theta, \phi, \rho_{\alpha \beta}^{2}\right)
$$

where

$$
\begin{aligned}
& W^{0}\left(\cos \theta, \phi, \rho_{\alpha \beta}^{0}\right)=\frac{3}{4 \pi}\left[\frac{1}{2} \sin ^{2} \theta+\frac{1}{2}\left(3 \cos ^{2} \theta-1\right) \rho_{00}^{0}-\sqrt{2} \operatorname{Re} \rho_{10}^{0} \sin 2 \theta \cos \phi-\rho_{1-1}^{0} \sin ^{2} \theta \cos 2 \phi\right] \\
& W^{1}\left(\cos \theta, \phi, \rho_{\alpha \beta}^{1}\right)=\frac{3}{4 \pi}\left[\rho_{11}^{1} \sin ^{2} \theta+\rho_{00}^{1} \cos ^{2} \theta-\sqrt{2} \operatorname{Re} \rho_{10}^{1} \sin 2 \theta \cos \phi-\rho_{1-1}^{1} \sin ^{2} \theta \cos 2 \phi\right] \\
& W^{2}\left(\cos \theta, \phi, \rho_{\alpha \beta}^{2}\right)=\frac{3}{4 \pi}\left[\sqrt{2} \operatorname{Im} \rho_{10}^{2} \sin 2 \theta \sin \phi+\operatorname{Im} \rho_{1-1}^{2} \sin ^{2} \theta \sin 2 \phi\right]
\end{aligned}
$$

Here, $\theta$ and $\phi$ are the polar and azimuthal angles of the $K^{+}\left(K^{-}\right)$decay product in the helicity reference frame ${ }^{1}$ and $P_{\gamma}$ is the degree of linear polarization of the photon beam. If the process is $s$-channel helicity conserving as delineated in VDM, then only two of the nine spin density matrix elements will prove to be nonzero, $\rho_{1-1}^{1}=0.5$ and $\operatorname{Im} \rho_{1-1}^{2}=-0.5$. Should there be any significant deviations from these values, this will clearly indicate mechanisms other than diffraction are present: that is new physics, such as $s \bar{s}$ knockout or baryon resonances decaying through the $\phi$ channel [7].

\section{PRELIMINARY REPORT ON THE $\phi$ MESON FROM G8B}

At the time of this writing we have just finished our first pass through compressing the entire dataset of the nine-week run. Our calibrations are good as evidenced by a very high degree of polarization $(\sim 75 \%)$ as in Fig. $2 b$ and the quality of the data as shown in Fig. 3b, where we can cleanly distinguish kaons from pions. For our first pass through the dataset, we make rather loose cuts on the particle masses, ${ }^{2}$ timing cuts in vertex time differences between particles and the incoming photon [13], and a loose $K^{-}$ missing mass cut. We primarily examine the $\vec{\gamma} p \rightarrow\left(p K^{+}\right) K^{-}$topology which gives the highest acceptance, since the low-momentum $K^{-}$mesons tend to bend away and not be detected in CLAS. We make further cuts for optimizing the vertex time differences between $\gamma$ and $p\left(\Delta t_{p, \gamma}\right)$ and $\gamma$ and $K^{+}\left(\Delta t_{K^{+}, \gamma}\right)$ in the target due to the 2-ns structure ${ }^{3}$ as shown in Fig. 3a, i.e. we accept events where $\left|\Delta t_{p, \gamma}\right|<2 \mathrm{~ns}$ and $\left|\Delta t_{p, \gamma}\right|<2 \mathrm{~ns}$. Another improvement of $\Delta t_{p, \gamma}$ is made by binning in momentum by steps of $0.08 \mathrm{GeV} / \mathrm{c}$; every single momentum bin is fit by a Gaussian function, whereby we extract the resolution $(\sigma)$ and peak position for making the appropriate $3-\sigma$ timing cut. A major component of

\footnotetext{
1 The $z$ - or quantization- axis is defined as the direction opposite the proton in the total c.m. system. Once the $z$-axis is so defined we then boost to the $\phi$ rest frame, wherein the direction of the decay $K^{+}$in the $\phi$-meson rest frame defines the polar angle $\theta$ and the azimuthal angle $\phi$ (with respect to the production plane); $\Phi$ is the angle between the photon polarization vector and the production plane.

${ }^{2}$ Here we make the cuts $0.3<M_{K^{+}}<0.7 \mathrm{GeV} / \mathrm{c}^{2}$ and $0.8<M_{p}<1.2 \mathrm{GeV} / \mathrm{c}^{2}$ for the $K^{+}$and proton.

3 Electrons bunched in buckets separated by $2 \mathrm{~ns}$ are directed onto the diamond radiator, producing one or more of photons within this 2-ns structure. A physical event is one where a difference between proton (kaon) time vertex must be separated from photon time vertex by less than $2 \mathrm{~ns}$.
} 

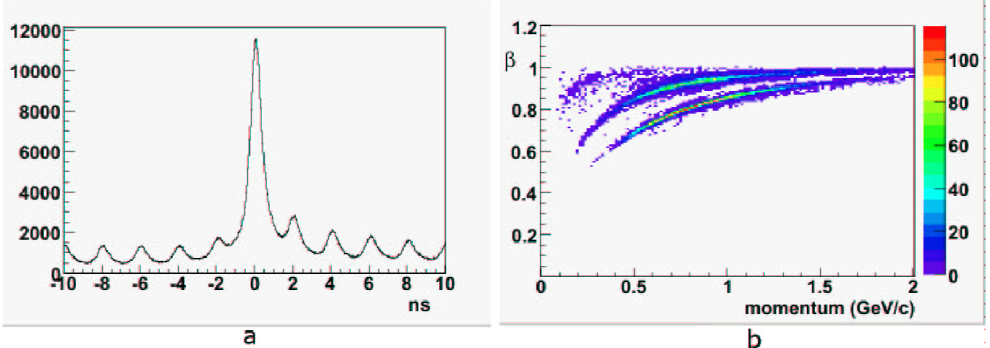

FIGURE 3. a) $\Delta t_{p, \gamma}$ timing between the proton start time and the photon time in the tagger. The 2 -ns structure reflects the 2 -ns rf bunching of the electrons in the accelerator. b) Particle reconstruction after cuts on the timing and the $K^{-}$missing mass. Here $\beta=\sqrt{\frac{p^{2}}{p^{2}+m^{2}}}$.

the background comes from non- $p K^{+} K^{-}$final states, which is then rejected by invoking a $K^{-}$missing mass cut as shown in Fig. 4. The result of $K^{+} K^{-}$invariant mass is plotted in Fig. 5 where the $1.020 \mathrm{GeV} / \mathrm{c}^{2} \phi$-meson peak rises high above the background.

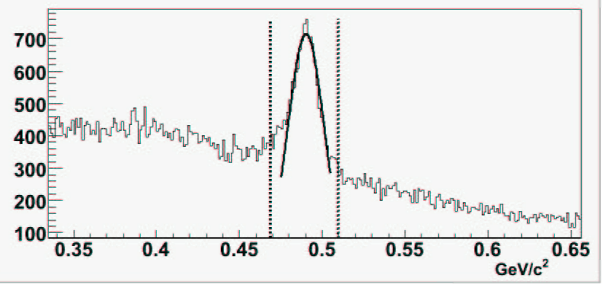

FIGURE 4. $K^{-}$missing mass reconstructed from $\vec{\gamma} p \rightarrow\left(p K^{+}\right) K^{-}$mode. The dashed lines bracket the peak around the $K^{-}$nominal mass, which is determined by the Gaussian fit. A 3- $\sigma$ mass cut then is made to separate the $K^{-}$candidates, i.e. $0.47<M_{K^{-}}<0.51 \mathrm{GeV} / \mathrm{c}^{2}$.

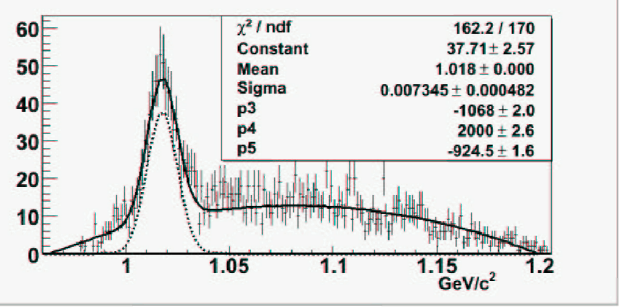

FIGURE 5. $K^{+} K^{-}$invariant mass. This represents $8 \%$ of the entire data for $1.9<E_{\vec{\gamma}}<2.1 \mathrm{GeV}$. From the fit, which is a combination of a Gaussian function and a second-order polynomial, we find that $\sim 550$ $\phi$ events are contained within the gaussian signal peak, with the signal to background being nearly $3: 1$.

In comparison with [14], there are just 53 events in the range between 2.4 to $3.3 \mathrm{GeV}$, which marked the world's data near the baryon resonance regime produced with linearlypolarized photons before the advent of SPring-8 [15]. We note that SPring- 8 and $g 8 b$ 
are complementary experiments, for whereas SPring- 8 has excellent acceptance for $|t|<0.4(\mathrm{GeV} / \mathrm{c})^{2}$ and little acceptance in the central and back polar angle regions, the reverse holds true for $\mathrm{g} 8 \mathrm{~b}$. This means $\mathrm{g} 8 \mathrm{~b}$ is sensitive to non-diffractive behavior for $|t|>0.5(\mathrm{GeV} / \mathrm{c})^{2}$.

\section{OUTLOOK}

We expect to enhance our event selection criteria by improving our $\Delta t_{\gamma, K^{+}}$cuts as a function of the $K^{+}$momentum and applying further momentum-dependent $E$ loss corrections. From five g8b runs, moreover, we collected approximately $550 \phi$ mesons, which represents $\sim 8 \%$ of the data sample for $1.9<E_{\vec{\gamma}}<2.1 \mathrm{GeV}$. This number is to be compared to the SPring-8 data sample of $5000 \phi$ mesons at low $|t|$. We expect to extract, then, well over $7500 \phi$ mesons from the entire g8b data set once we fold the g8b data from $1.7<E_{\vec{\gamma}}<1.9 \mathrm{GeV}$. This means we will be able to bin the decay angular distribution of the $\phi$ meson and thereby extract the aforementioned density matrix elements as functions of the center of mass energy squared, $s$, and four momentum transfer squared, $t$.

\section{ACKNOWLEDGMENTS}

This work was made possible through a grant from the National Science Foundation, NSF-0555497. And, in particular, we thank our colleagues at Glasgow University, Arizona State University, and The Catholic University of America, who played a key role in making g8b a success. We also wish to thank Dr. Tsutomu Mibe for his constructive comments on this paper.

\section{REFERENCES}

1. R.A. Williams, Phys. Rev. C 57, 223 (1998).

2. T. Mibe, "Measurement of $\phi$-meson photoproduction near production threshold with linearly polarized photons," Ph.D. Thesis, Osaka University, Japan (2004), unpublished. T. Mibe et al. Phys. Rev. Lett. 95, 182001 (2005).

3. E.M. Henley, G. Krein, and A.G. Williams, Phys. Lett. B 281, 178 (1992).

4. A.I. Titov, Y. Oh, S.N. Yang, and T. Morii, Phys. Rev. C 58, 2429 (1998); Nucl. Phys. A 684, 354 (2001).

5. A.I. Titov and T.-S.H. Lee, Phys. Rev. C 67, 065205 (2003).

6. Q. Zhao, B. Saghai, and J.S. Al-Khalili, Phys. Lett. B 509, 231 (2001).

7. P.L. Cole, J. Mueller, D.J. Tedeschi et al. "Photoproduction of $\phi$ Mesons with Linearly Polarized Photons," JLab Experiment E-98-109.

8. P.L. Cole, J. Kellie, F.J. Klein, K. Livingston, J.A. Mueller, J.C. Sanabria, and D.J. Tedeschi, EMI2001, (World Scientific) ISBN 981-238-044-2. (2002).

9. B.A. Mecking et al., Nucl. Instrum. and Methods A 503/3, 513 (2003).

10. K. Schilling, P. Seyboth, and G. Wolf, Nucl. Phys. B 15, 397 (1970).

11. B.A. Robson, "The Theory of Polarization Phenomena," Clarendon Press, Oxford (1974).

12. M. Jacob and G.C. Wick, Ann. Phys. 7, 404 (1959).

13. T. Mibe, H. Gao, K. Hicks, K. Kramer, S. Stepanyan, and D. Tedeschi, "Coherent $\phi-m e s o n$ photoproduction on deuterium," CLAS-Analysis Note: 2007-1 10,9 October 2006.

14. J. Ballam et al., Phys. Rev. D 7, 3150 (1973).

15. T. Nakano et al., Nucl. Phys. A 684, 71 (2001). 